\title{
Exactly solvable tight-binding models on two scale-free networks with identical degree distribution
}

\author{
Pinchen $\mathrm{Xie} *$ \\ Department of Physics, Fudan University, Shanghai 200433, China and \\ Shanghai Key Laboratory of Intelligent Information Processing, Fudan University, Shanghai 200433, China \\ Bo $\mathrm{Wu}$ \\ Department of Applied Mathematics and Statistics, \\ Stony Brook University, Stony Brook, NY 11794, USA \\ Zhongzhi Zhandi \\ Shanghai Key Laboratory of Intelligent Information Processing, Fudan University, Shanghai 200433, China and \\ School of Computer Science, Fudan University, Shanghai 200433, China
}

(Dated: September 14, 2018)

\begin{abstract}
We study ideal Bose gas upon two scale-free structures with identical degree distribution. Energy spectra belonging to tight-binding Hamiltonian are exactly solved and the related spectral dimensions of $\mathcal{G}^{1}$ and $\mathcal{G}^{2}$ are obtained as $d_{s_{1}}=2$ and $d_{s_{2}}=2 \ln 4 / \ln 3$. We show Bose-Einstein condensation will only take place upon $\mathcal{G}^{2}$ instead of $\mathcal{G}^{1}$. The topology and thermodynamical property of the two structures are proven to be totally different.
\end{abstract}

\section{INTRODUCTION}

Understanding the spectral structure of Hamiltonians is of key importance in the study of Bose-Einstein condensation (BEC). The underlying dimensionality of a spectrum determines the occurrence of BEC in most cases. It is well known that for ideal and uniform (homogenous) Bose gases, only the energy spectra related to three or higher dimensions allow Bose-Einstein phase transition [1]. For confined (inhomogenous) Bose gases, it was shown the presence of traps allow BEC to take place in lower dimension notwithstanding 2, 3]. Obviously, the structure and magnitude of present traps have large influence on the spectral structure for quantum gases, and alter the phase-transition phenomena as well. To further show how the structure of traps influences the thermodynamic behaviors of confined quantum gases, several types of weakly-coupled discrete traps are investigated over the past decade with the help of network theory [4-10]. For these Bose gases confined by traps with network-like structure, the displayed BEC is topology-induced. For example, BEC on star-shaped and wheel-shaped network depends on the number of star-arms and wheel spokes [4, 6]. The type of Bose-Einstein transition gone through upon diamond hierarchical lattices is also shown to be fully determined by a structural parameter of the lattice-like trap [7]. Moreover, a fractal-like energy spectrum is found in Bose gas confined by Apollonian network-shaped traps, which shows self-similarity at the same time [10]. As we can see, the topology of different kinds of traps, usually embedded in a $n$-dimensional Euclidean space, is a key issue to studying condensation of inhomogenously confined Bose gas. However, still no theory can give a satisfactory explanation how the structures of trap distributions interact with the spectral structure and determine BEC phenomenon. It is also unclear what topological invariant can decide the occurrence of BEC or the type of phase transition. To fill this gap, we pay our attention to traps with scale-free topology. We will determine whether the scale-free characteristics of weakly coupled traps will govern the BEC.

The term "scale-free" mentioned before is used to describe a network with a power-law degree distribution. In recent decades, dynamics of inhomogenously coupled systems with a scale-free topology have been studied extensively since lots of real-world networks were verified to inherit the same nature [8, 9, 11 24]. The Hamiltonian defined by coupling between adjacent vertices is used to describe the time evolution of such systems, related to many observable phenomena [10, 25 27]. The entailed dynamics are highly dependent on the network topology, as the BEC we focus on in this paper. Among all the topological features of networks, the degree distribution is one of the most important characteristics. Many networks with a scale-free degree distribution have fractal-like properties, due to the selfsimilarity underlying its topological structure 28 31. It was also reported that the scale-free characteristics control the critical phenomena of many physical processes [15, 20 22]. Meanwhile, some literature suggests that scale-free

*Electronic address: xiepc14@fudan.edu.cn

${ }^{\dagger}$ Electronic address: zhangzz@fudan.edu.cn URL: http://www.researcherid.com/rid/G-5522-2011 
characteristics will not determine phase transitions related to cooperative behaviors and epidemic spreadings [23, 24]. Nevertheless we will show BEC upon weakly coupled traps belongs to the latter.

In this study, we construct two scale-free networks sharing the same degree distribution. We introduce the tightbinding Hamiltonian to describe weakly coupled traps [4 [10]. We solve the fractal-like spectra exactly for both networks. And their spectral dimensions are analytically calculated [32, 33]. The condensed fraction of low-temperature ideal Bose gas in thermodynamic limit is computed numerically. And we find these two structures display totally different BEC phenomena.

\section{CONSTRUCTION}

We construct two scale-free networks iteratively in two different patterns (see Fig 1). The first few iterations are shown in Fig 2. We label them as $\mathcal{G}_{t}^{1}$ and $\mathcal{G}_{t}^{2}$ respectively according to the pattern (1 or 2) and iterations $(t \in N)$ they undergo. $\mathcal{G}^{1}$ and $\mathcal{G}^{2}$ denote the networks after infinitely many iterations $(t \rightarrow \infty)$.

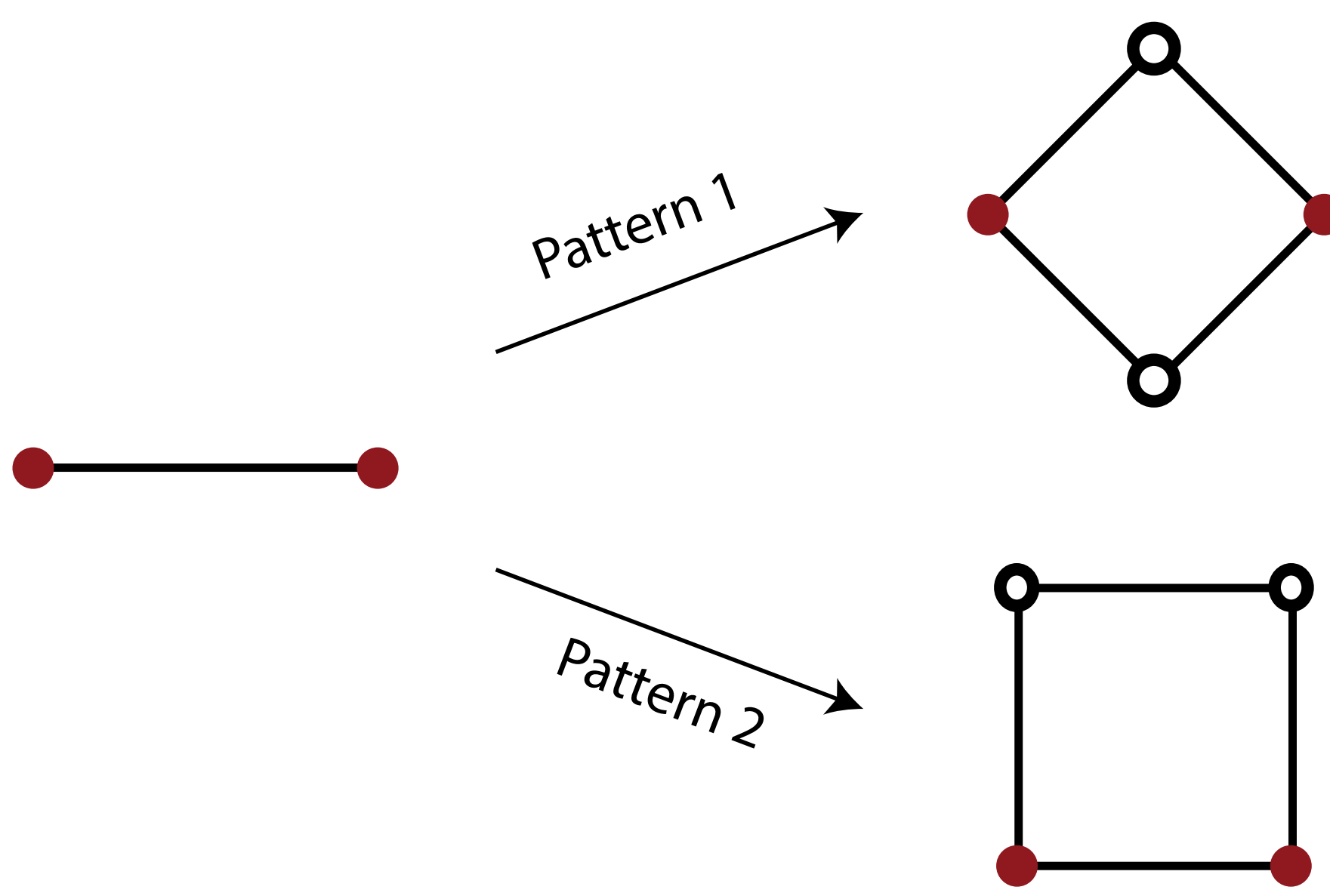

FIG. 1: (Color online) Two patterns of construction.

The degree of a vertex is defined as the number of its adjacent vertices. For both patterns of iteration, the degree of any vertex is doubled after one iteration. Thus, $\mathcal{G}_{t}^{1}$ and $\mathcal{G}_{t}^{2}$ have the same degree distribution $P(k)$, that is the probability distribution of the degrees of vertices over the whole network. After some algebra, we obtained several common characteristics of the two structures. The total number of edges is $E_{t}=4^{t}$ for both $\mathcal{G}_{t}^{1}$ and $\mathcal{G}_{t}^{2}$ and the total number of vertices is $N_{t}=\frac{2}{3}\left(4^{t}+2\right)$. Their degree distributions simultaneously obey $P(k) \sim k^{-3}$ when $t \rightarrow \infty$.

The power-law behavior of $P(k)$ indicates $\mathcal{G}^{1}$ and $\mathcal{G}^{2}$ are both scale-free networks. But $\mathcal{G}^{1}$ and $\mathcal{G}^{2}$ are clustered differently. Let $L$ denote the typical distance (length of the shortest path) between two randomly chosen nodes of a network. For $\mathcal{G}_{t}^{2}$,

$$
L \sim \log N_{t} \sim t
$$




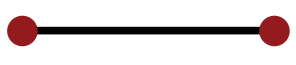

$t=0$

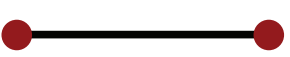

$t=0$
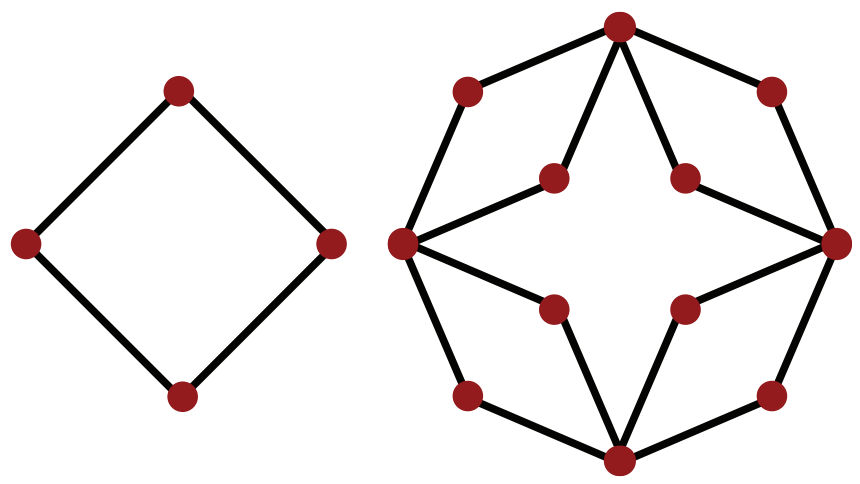

$t=2$
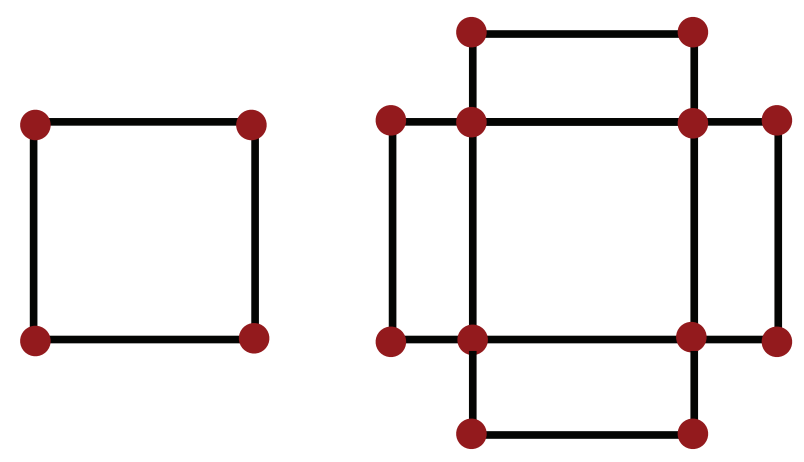

$t=2$

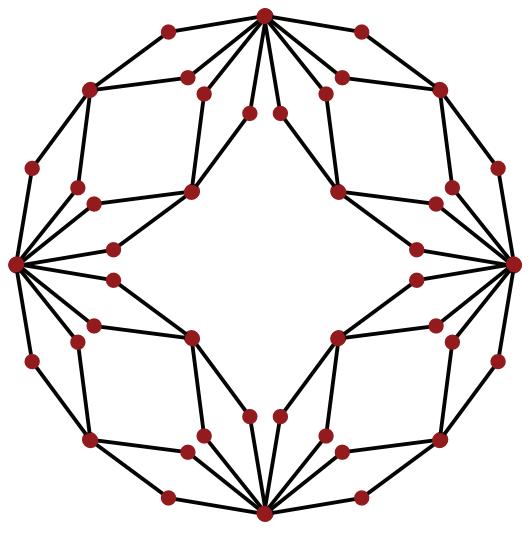

$t=3$

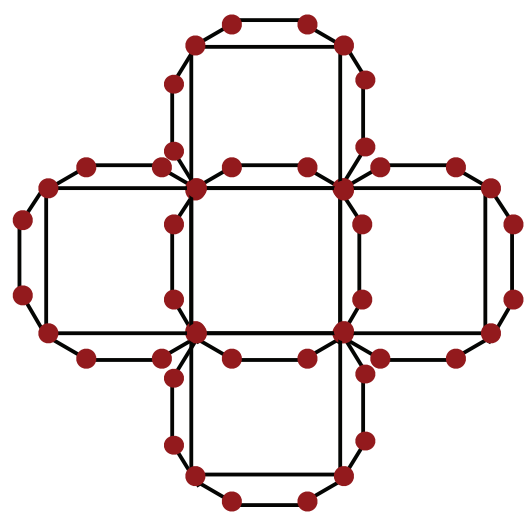

$t=3$

FIG. 2: (Color online) The first three iterations.

holds for large $t$. This means $\mathcal{G}^{2}$ is a small-world network [34] with a high clustering coefficient [35], while $\mathcal{G}_{t}^{1}$ is not since its average shortest path length grows faster than $\log N_{t}$.

Evidently, the degree distribution does not determine all the global property of a scale-free network. More information is encoded in the pattern the local vertices are organized.

\section{TIGHT-BINDING BOSONS ON INHOMOGENEOUS STRUCTURES}

\section{A. Tight-binding Hamiltonian}

Label the vertices of any connected graph $\mathcal{G}=(\mathcal{V}, \mathcal{E})(\mathcal{V}$ is the set of vertices and $\mathcal{E}$ the set of edges) from 1 to $N$. Label the edge connecting vertex $i$ and $j$ by $e_{i j}$. Suppose $G$ corresponds to a network of weakly coupled traps, where vertex denotes trap, edge denotes coupling. Following the tight-binding model defined in [10], which is also a simplified LCAO model [36], the Hamiltonian for non-interacting bosons on $\mathcal{G}$ is

$$
H=\sum_{k \in V} \epsilon_{k}|k\rangle\left\langle k\left|+\sum_{i, j \in V} h_{i j}\right| i\right\rangle\langle j|
$$

where $|k\rangle$ is the orbit of localized boson at vertex $k$ and $\epsilon_{k}$ the on-site ground energy. We ignore all excited states. $h_{i j}$ is the hopping amplitude between trap (vertex) $i$ and $j$. Here we only consider the overlaps of localized states corresponding to adjacent vertices. So $h_{i j}=0$ if vertex $i$ is not connected to vertex $j$. Suppose the on-site energy is uniform among all sites, we put $\epsilon_{k}=0$ in Eq. (2) without loss of generality. 
Next, let us introduce some algebra tools for formulating our model.

Adjacency matrix $A$ is used to describe the connection among vertices of graph $\mathcal{G}$ :

$$
A_{i j}= \begin{cases}1 & e_{i j} \in E \\ 0 & e_{i j} \notin E\end{cases}
$$

The degree matrix $D$ of $\mathcal{G}$ is diagonal, given as $D=\operatorname{diag}\left(d_{1}, d_{2}, \cdots, d_{N}\right)$ where $d_{k}$ is the degree of vertex $k$.

The transition matrix $M$ of the same graph is defined as $M=D^{-1} A$, which can be normalized as $P=D^{\frac{1}{2}} M D^{-\frac{1}{2}}=$ $D^{-\frac{1}{2}} A D^{-\frac{1}{2}}$. The element of $P$ is thus $P_{i j}=\frac{1}{\sqrt{d_{i} d_{j}}}$.

For homogenous systems such as Bravais lattices, the hopping amplitude is usually taken as $h_{i j}=\xi A_{i j}$, where $\xi$ is constant. In the case of scale-free structures, we assume $h_{i j}=\xi P_{i j}$ to avoid divergence difficulty.

Further, we introduce the reduced Hamiltonian $\mathcal{H}=\frac{1}{\xi} H$. Eq. (2) can be rephrased as

$$
\mathcal{H}=\sum_{i, j \in V} \frac{1}{\sqrt{d_{i} d_{j}}}|i\rangle\langle j|
$$

\section{B. Complete energy spectra}

Next, we will solve the energy spectrum of $\mathcal{H}$ for $\mathcal{G}^{1}$ and $\mathcal{G}^{2}$ by exact matrix renormalization. Similar derivation of spectrum for other renormalizable structures can be found at [37, 38].

Let $D_{i, t}$ and $A_{i, t}$ denote the degree matrix and adjacency matrix of $\mathcal{G}_{t}^{i}(i=1,2)$.

For $i=1$, by proper permutation of rows and columns, $D_{i, t}$ and $A_{i, t}$ write

$$
\begin{gathered}
D_{1, t}=\left(\begin{array}{cc}
2 I & 0 \\
0 & 2 D_{1, t-1}
\end{array}\right), \\
A_{1, t}=\left(\begin{array}{cc}
0 & J^{T} \\
J & 0
\end{array}\right)
\end{gathered}
$$

where $I$ is the identity matrix of order $\Delta_{t}=N_{t}-N_{t-1}$. And $N_{t-1} \times \Delta_{t}$ matrix $J$ represents the adjacency among new vertices and the old ones. The hopping matrix (i.e. the normalized transition matrix of $\mathcal{G}_{t}^{1}$ ) is

$$
P_{1, t}=D_{1, t}^{-\frac{1}{2}} A_{1, t} D_{1, t}^{-\frac{1}{2}}=\left(\begin{array}{cc}
0 & \frac{1}{2} J^{T} D_{1, t-1}^{-\frac{1}{2}} \\
\frac{1}{2} D_{1, t-1}^{-\frac{1}{2}} J & 0
\end{array}\right) .
$$

The characteristic polynomial of $P_{t}^{1}$ is thus

$$
\operatorname{det}\left(\lambda-P_{1, t}\right)=\lambda^{N_{t}-N_{t-1}} \operatorname{det}\left(\lambda-\frac{1}{4 \lambda} D_{1, t-1}^{-\frac{1}{2}} J J^{T} D_{1, t-1}^{-\frac{1}{2}}\right),
$$

using the identity

$$
\operatorname{det}\left(\begin{array}{cc}
A & B \\
C & D
\end{array}\right)=\operatorname{det} A \cdot \operatorname{det}\left(D-C A^{-1} B\right)
$$

Let $j_{m n}$ denote the $(m, n)$-entry of $J$. The symmetric matrix $J J^{T}$ is represented by

$$
\left(J J^{T}\right)_{m n}=\sum_{l} j_{m l} j_{n l}
$$

For $m=n,\left(J J^{T}\right)_{m n}$ is exactly the degree of node $m$ in $\mathcal{G}_{t}^{1}$, i.e., twice its degree in $\mathcal{G}_{t-1}^{1}$. For $m \neq n,\left(J J^{T}\right)_{m n}$ is 2 if node $m$ and node $n$ is previously adjacent in $\mathcal{G}_{t-1}^{1}$, otherwise 0 .

Hence one obtains

$$
J J^{T}=2 D_{1, t-1}+2 A_{1, t-1},
$$




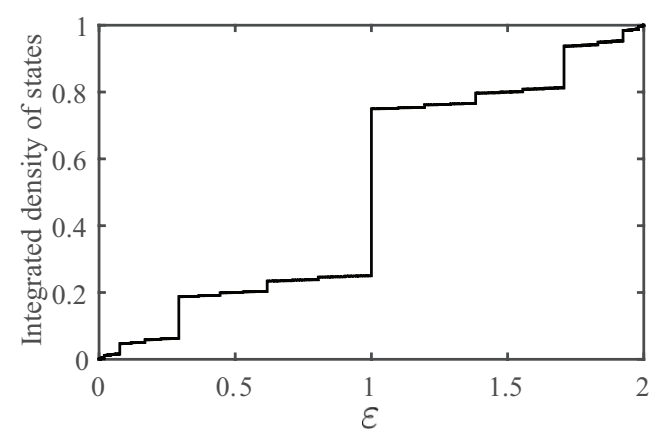

FIG. 3: The fractal-like integrated density of states for $\mathcal{G}_{12}^{1}$.

which leads to

$$
\operatorname{det}\left(\lambda-P_{1, t}\right)=\frac{1}{2}^{N_{t-1}} \lambda^{N_{t}-2 N_{t-1}} \operatorname{det}\left(\left(2 \lambda^{2}-1\right)-P_{1, t-1}\right) .
$$

The recursive relation Eq. (12) indicates, if $\lambda$ is an eigenvalue of $P_{1, t}$, then $R_{1}(\lambda)=\left(2 \lambda^{2}-1\right)$ is an eigenvalue of $P_{1, t-1}$ with the same degeneracy unless $\lambda=0$. Conversely, for any eigenvalue $\eta$ of $P_{1, t-1}$, the inverse of $R_{1}$ gives its two descendents with the same degeneracy as $\eta$, unless $\eta=-1$ (notice $R_{1}(-1)=0$ ). Hence the degeneracy of the exceptional eigenvalue 0 should be $\frac{N_{t}}{2}$ to ensure the spectrum of $P_{1, t-1}$ is complete.

Subsequently, the spectrum of $P_{1, t}^{2}$, denoted by $\sigma_{1, t}$, can be analytically determined from the initial spectrum $\sigma_{1,1}=\left\{\cos 0, \cos \frac{\pi}{2}, \cos \frac{\pi}{2}, \cos \pi\right\}$, given as

$$
\sigma_{1, t}=\left\{E_{i}\right\}=\bigcup_{\substack{0 \leqslant k \leqslant 2^{r} \\ 0 \leqslant r \leqslant t}}\left\{\cos \frac{k \pi}{2^{r}}\right\} .
$$

The symmetry of $\sigma_{1, t}$ with respect to 0 is obvious and the lowest energy is always $E_{0}=-1$.

The integrated density of states (IDOS) $f(\varepsilon)$ is defined to be the number of states between $E_{0}$ and $E_{0}+\varepsilon(0 \leq \varepsilon \leq 2)$ divided by the total number of states.

For homogenous systems such as a single particle in a 3-D cavity, it is well known that $f(\varepsilon) \propto \varepsilon^{\frac{3}{2}}$. For inhomogenous systems also showing power-law behaviors $f(\varepsilon) \propto \varepsilon^{d_{s} / 2}$ near the band bottom $(\varepsilon \ll 1)$, the exponent $d_{s} / 2$ can be irrational. $d_{s}$ is said to be the spectral dimension of a structure related to certain Hamiltonian [32, 33]. It was reported that spectral dimension is a crucial index categorizing the universality classes of topology-induced BoseEinstein transitions [7]. Also, it has been proven that the phase transition breaking a continuous symmetry can not take place on systems with a spectral dimension not greater than 2 [39 42]. The theoretical determination of spectral dimension is not an easy task. However, by appropriate renormalization, analytical results on spectral dimension are still obtainable for several fractal-like structures [43].

To compute the spectral dimension of our networks, we first pay attention to the IDOS $f(\varepsilon)$ corresponding to $\mathcal{G}^{1}$. Transforming $R_{1}$ for expressing the iterative relation for the relative energy $\varepsilon$, one obtains $\tilde{R}_{1}(\varepsilon)=2(\varepsilon-1)^{2}$. The following relation holds for small $\varepsilon$ because $\mathcal{G}^{1}$ is invariant under iteration :

$$
\begin{aligned}
f(\varepsilon) & =\left(1-f\left(\tilde{R}_{1}(\varepsilon)\right)\right) \lim _{t \rightarrow \infty} \frac{N_{t-1}}{N_{t}} \\
& =\frac{1}{4} f\left(2-\tilde{R}_{1}(\varepsilon)\right) \\
& \approx \frac{1}{4} f(4 \varepsilon) .
\end{aligned}
$$

Hence $f(\varepsilon) \propto \varepsilon^{1}$ near the origin. The spectral dimension of $\mathcal{G}^{1}$ is thus $d_{s_{1}}=2$. Fig. 3 gives schematic representation of the spectrum.

By the same method, we calculate the spectrum for $\mathcal{G}_{t}^{2}$ and obtain the iterative expression for the characteristic polynomial:

$$
\begin{aligned}
\operatorname{det}\left(\lambda-P_{2, t}\right)= & 2^{-N_{t-1}} \cdot\left(\lambda^{2}-\frac{1}{4}\right)^{\frac{N_{t}-3 N_{t-1}}{2}} \\
& \cdot \lambda^{2 N_{t-1}} \cdot \operatorname{det}\left(\frac{2 \lambda^{2}-1}{\lambda}-P_{2, t-1}\right) .
\end{aligned}
$$




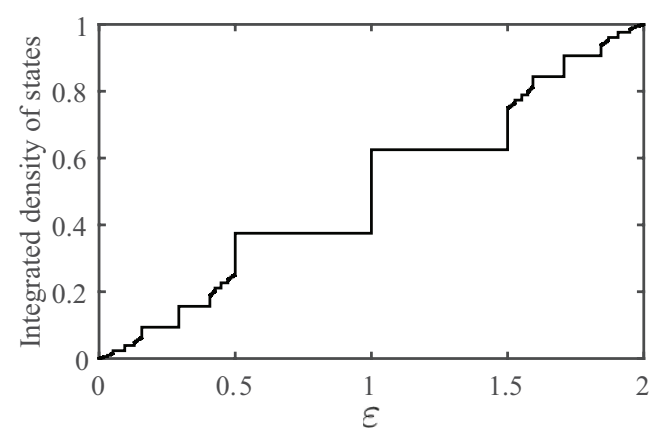

FIG. 4: The integrated density of states for $\mathcal{G}_{12}^{2}$.

Now the iteration is formulated as $R_{2}(\lambda)=\frac{2 \lambda^{2}-1}{\lambda}$. By taking the inverse of $R_{2}$, we are able to generate the spectrum of any order from the initial one. The exceptional eigenvalues here are $\frac{1}{2},-\frac{1}{2}$ and 0 . Their degeneracies are $\left(N_{t}-\right.$ $\left.3 N_{t-1}+2\right) / 2,\left(N_{t}-3 N_{t-1}+2\right) / 2$ and $N_{t-1}$ respectively according to Eq. (15). Since $\sigma_{2,1}=\{1,0,0,-1\}$, the energy spectrum $\sigma_{2, t}$ for $\mathcal{G}_{t}^{2}$ is symmetric with respect to zero and the lowest-energy is still $E_{0}=-1$. For $\mathcal{G}^{2}$, when $\varepsilon$ is small, the invariance of $f(\varepsilon)$ requires

$$
\begin{aligned}
f(\varepsilon) & =f\left(2 \varepsilon-1-\frac{1}{\varepsilon-1}\right) \lim _{t \rightarrow \infty} \frac{N_{t-1}}{N_{t}} \\
& \approx \frac{1}{4} f(3 \varepsilon) .
\end{aligned}
$$

Thus

$$
f(\varepsilon) \propto \varepsilon^{\frac{d_{s_{2}}}{2}}
$$

near the band bottom and the spectral dimension is

$$
d_{s_{2}}=2 \frac{\ln 4}{\ln 3}=2.524 .
$$

The IDOS for finite-size network is shown in Fig. 4

By so far, the spectra related to the two networks have shown unique fractal-like structures, which will leads to different behaviors of tight-binding particles.

\section{DIFFERENT CRYOGENIC BEHAVIORS OF BOSE GAS}

In this section we investigate the cryogenic behaviors of non-interacting Bose gas on $\mathcal{G}^{1}$ and $\mathcal{G}^{2}$ and check whether Bose-Einstein condensation will take place.

Suppose there are $N_{p}$ bosons on the structures. The particle density is defined as $\gamma=\frac{N_{p}}{N_{t}}$. To approach the thermodynamic limit, we fix $\gamma$ and let $t \rightarrow \infty$. Bose-Einstein statistics gives the expected number of bosons in state $i$ :

$$
n_{i}=\frac{1}{z^{-1} e^{\beta \xi \varepsilon}-1}
$$

where $\beta=\frac{1}{k_{B} T}$ and the fugacity $z=e^{\beta\left(\mu-\xi E_{0}\right)} \cdot \mu$ is the chemical potential.

$$
n_{0}=\frac{1}{z^{-1}-1}
$$

is the number of condensed particles and $f=\frac{n_{0}}{\gamma N_{t}}$ the condensed fraction.

The normalization condition requires

$$
\sum_{i} n_{i}=\gamma N_{t}
$$




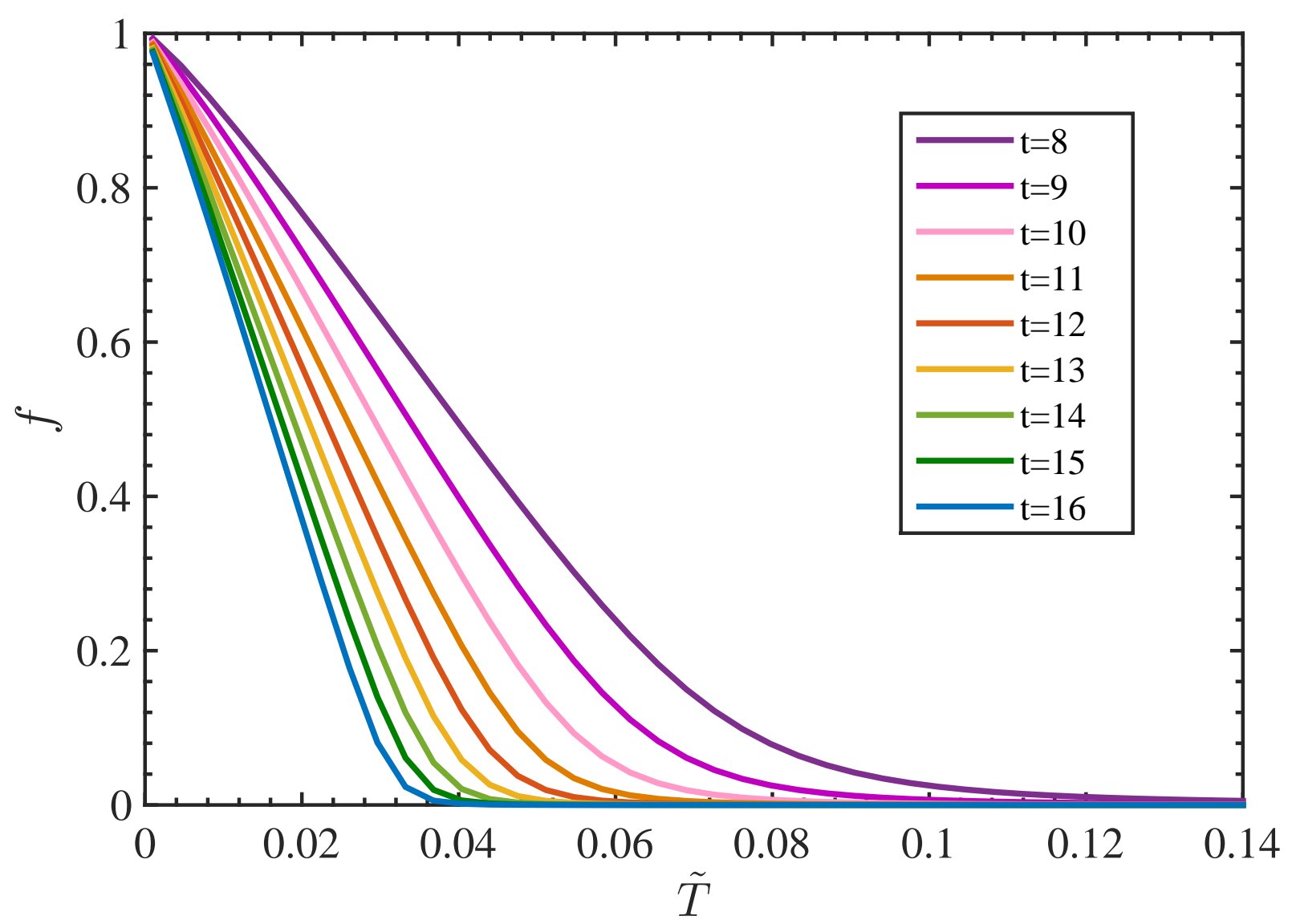

FIG. 5: (Color online) Condensed fraction $f$ as a function of $\tilde{T}$ for $G_{t}^{1}(\gamma=0.2)$. The finite-size effect decreases as $t$ increases.

Transforming Eq. (21) into integral, one obtains

$$
\int_{0}^{2} \frac{\rho(\varepsilon)}{z^{-1} e^{\beta \xi \varepsilon}-1} d \varepsilon=\gamma
$$

where $\rho(\cdot)$ is the states density and $\varepsilon$ the relative energy.

Let $\tilde{T}=\frac{k_{B} T}{\xi}$ be the dimensionless temperature. Substitute $\xi \beta$ with $\tilde{\beta}=1 / \tilde{T}$. Eq. (22) is rewritten as

$$
\int_{0}^{2} \frac{\rho(\varepsilon)}{z^{-1} e^{\tilde{\beta} \varepsilon}-1} d \varepsilon=\gamma
$$

Below the critical temperature where the Bose-Einstein phase-transition occurs, $z$ is always 1 . In Fig. [5 we present the relation between the condensed fraction and the dimensionless temperature with finite-size effects. There is no sign of first order phase-transition, which is proved analytically as follows.

Suppose $\mathcal{G}^{1}$ allows the occurrence of BEC transition in thermodynamic limit. The uncondensed fraction of bosons at the (dimensionless) critical temperature $T_{c_{1}}$ is

$$
f_{u}=\frac{1}{\gamma} \lim _{\epsilon \rightarrow 0} \int_{\epsilon}^{2} \frac{\rho(\varepsilon)}{e^{\beta_{c_{1}} \varepsilon}-1} d \varepsilon>\frac{1}{\gamma} \int_{\epsilon_{1}}^{\epsilon_{2}} \frac{\rho(\varepsilon)}{e^{\beta_{c_{1}} \varepsilon}-1} d \varepsilon
$$

where $\beta_{c_{1}}=\frac{1}{T_{c_{1}}}, \epsilon_{1}<\epsilon_{2} \ll 1$. 


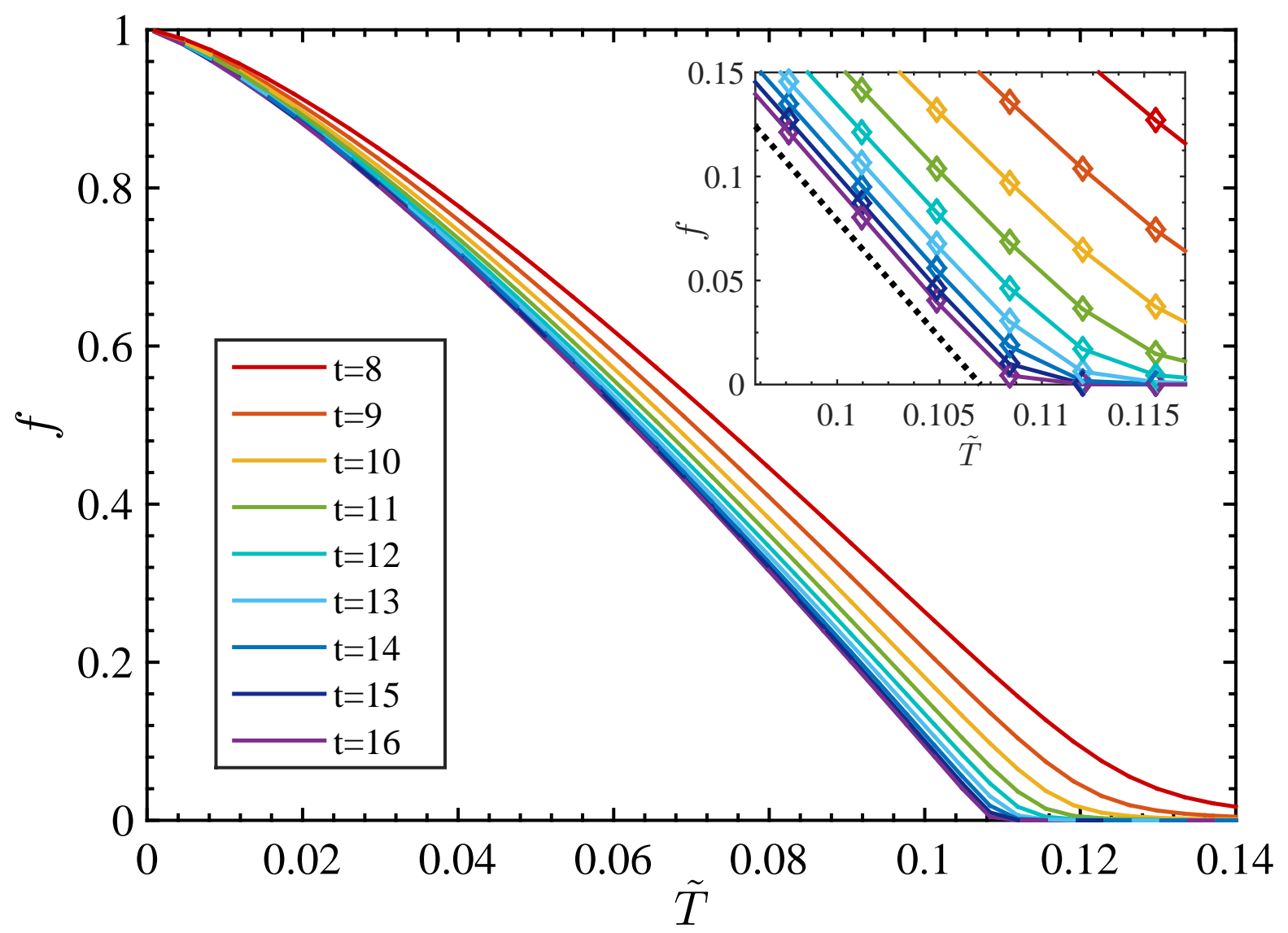

FIG. 6: (Color online) Condensed fraction $f$ as a function of $\tilde{T}$ for $\mathcal{G}_{t}^{2}(\gamma=0.2)$. The dotted line corresponds to the critical behavior.

By the approximation $\rho(\varepsilon) \approx k \frac{d\left(\epsilon \frac{d_{s_{1}}}{2}\right)}{d \epsilon}$, the last integral becomes

$$
\begin{aligned}
\frac{1}{\gamma} \int_{\epsilon_{1}}^{\epsilon_{2}} \frac{\rho(\varepsilon)}{e^{\beta_{c_{1}} \varepsilon}-1} d \varepsilon & \approx \frac{1}{\gamma} \int_{\epsilon_{1}}^{\epsilon_{2}} k \frac{d\left(\epsilon^{\frac{d_{s_{1}}}{2}}\right)}{d \epsilon} \cdot \frac{1}{e^{\beta_{c_{1}} \varepsilon}-1} d \varepsilon \\
& =\frac{k}{\gamma} \int_{\epsilon_{1}}^{\epsilon_{2}} \frac{1}{e^{\beta_{c_{1}} \varepsilon}-1} d \varepsilon
\end{aligned}
$$

where $k$ is a positive constant.

Since $\epsilon_{1}$ and $\epsilon_{2}$ are small, $e^{\beta_{c_{1}} \epsilon} \approx 1+\beta_{c_{1}} \epsilon$ holds for $\epsilon \in\left[\epsilon_{1}, \epsilon_{2}\right]$. It follows that

$$
\frac{k}{\gamma} \int_{\epsilon_{1}}^{\epsilon_{2}} \frac{1}{e^{\beta_{c_{1}} \varepsilon}-1} d \varepsilon \approx \frac{k}{\gamma \beta_{c_{1}}} \ln \left(\frac{\epsilon_{2}}{\epsilon_{1}}\right) .
$$

Thus,

$$
1 \geq f_{u}>\frac{k}{\gamma \beta_{c_{1}}} \ln \left(\frac{\epsilon_{2}}{\epsilon_{1}}\right) .
$$

$\epsilon_{2} / \epsilon_{1}$ has no finite upper bound. This contradicts Eq. 27. when $T_{c_{1}}>0$. Hence positive $T_{c_{1}}$ does not exist for $\mathcal{G}^{1}$. In consequence, Bose-Einstein condensation won't take place in low temperature.

Will the massive bosons behave differently on $\mathcal{G}^{2}$ in low temperature? A schematic representation of the relation between condensed fraction for network $\mathcal{G}^{2}$ and $\tilde{T}$ is given in Fig. 6. The curves converge quickly, suggesting the transition temperature when thermodynamic limit is approached. For $\gamma=0.2$, we obtain the transition temperature $\tilde{T}_{c} \approx 0.107$ and $f \propto\left|T-T_{c}\right|^{1}$ near the critical point. 
By further numerical computation, the dependence of the critical temperature on $\gamma$ and a series of critical exponents can be obtained, which are not the main interest of this study. Related discussions on these can be found at [7, 44]. We claim that the BEC in $\mathcal{G}^{2}$ belongs to the universality class of the ideal BEC in networks with spectral dimension $d_{s} \approx 2.524$.

Moreover, the state $\psi_{c}$ of the condensate can be determined analytically. Since hopping amplitude is negative $(\xi<0), \psi_{c}$ is related to the eigenvector of $P_{2, t}$ with respect to the eigenvalue 1 , which is usually called the equilibrium distribution or steady state of random walks depicted by the transition matrix. With finite-size effects $(t$ is finite), $\psi_{c}$ is expressed as

$$
\psi_{c}=A \sum_{i} \frac{d_{i}}{N_{t}}|i\rangle
$$

A is the normalization constant. The sum is taken over all the vertices.

Eq. (28) indicates the state of the condensate follows the degree distribution though the occurrence of phase transition is determined by more in-depth topology. Not only for $\mathcal{G}^{2}$, this consequence holds true for all discrete structures with a tight-binding Hamiltonian. If the structure is a random regular graph, the lowest state is simply the unweighted combination of all tight-binding local orbits up to phase difference.

\section{CONCLUSION}

Tight-binding models upon two scale-free networks with identical degree distribution $P(k) \sim k^{-3}$ were investigated.

By renormalization, we iteratively obtained the fractal-like spectra of the two networks and determined their spectral dimensions $\left(d_{s_{1}}=2, d_{s_{2}}=2 \ln 4 / \ln 3\right)$. Suggested by the value of $d_{s_{1}}$, we analytically proved BEC would not take place in $\mathcal{G}^{1}$. On the contrary, with the same scale-free degree distribution, the structure of $\mathcal{G}^{2}$ allows the occurrence of the Bose-Einstein phase-transition. Meanwhile, the BEC in $\mathcal{G}^{2}$ belongs to the universality class of the ideal BEC, related to spectral dimension $d_{s}=2 \ln 4 / \ln 3$. We also found the state $\psi_{c}$ for the condensate, which was determined by the degree distribution of the structure.

The divergent behaviors of the two structures give a good example how the topology as well as thermodynamical property of networks varies regardless of scale-free characteristics. The divergence not merely lies in several critical exponents but the occurrence of phase-transition. The scale-free characteristics do not always play a important role in dynamical systems governed by equations of the form $d x_{i} / d t=\sum k_{i j}\left(x_{j}-x_{i}\right)$, related to diffusion, relaxation, etc.

\section{Acknowledgments}

This work was supported by the National Natural Science Foundation of China under Grant No. 11275049.

[1] Kerson Huang. Statistical Mechanics. Wiley, 2 edition, 1987.

[2] Vanderlei Bagnato and Daniel Kleppner. Bose-Einstein condensation in low-dimensional traps. Phys. Rev. A, 44(11):74397441 , dec 1991.

[3] Wolfgang Ketterle and N. J. van Druten. Bose-Einstein condensation of a finite number of particles trapped in one or three dimensions. Phys. Rev. A, 54(1):656-660, jul 1996.

[4] I Brunelli, G Giusiano, F P Mancini, P Sodano, and A Trombettoni. Topology-induced spatial Bose-Einstein condensation for bosons on star-shaped optical networks. J. Phys. B At. Mol. Opt. Phys., 37(7):S275, 2004.

[5] P Buonsante, R Burioni, D Cassi, and A Vezzani. Bose-Einstein condensation on inhomogeneous networks: Mesoscopic aspects versus thermodynamic limit. Phys. Rev. B, 66(9):94207, 2002.

[6] EJGG Vidal, R P A Lima, and M L Lyra. Bose-Einstein condensation in the infinitely ramified star and wheel graphs. Phys. Rev. E, 83(6):61137, 2011.

[7] M L Lyra, FABF de Moura, I N de Oliveira, and M Serva. Bose-Einstein condensation in diamond hierarchical lattices. Phys. Rev. E, 89(5):52133, 2014.

[8] Maurizio Serva. Exactly solvable tight-binding model on the RAN: fractal energy spectrum and Bose-Einstein condensation. J. Stat. Mech. Theory Exp., 2014(8):P08018, 2014.

[9] I N De Oliveira, FABF De Moura, M L Lyra, J S Andrade Jr, and E L Albuquerque. Free-electron gas in the Apollonian network: Multifractal energy spectrum and its thermodynamic fingerprints. Phys. Rev. E, 79(1):16104, 2009.

[10] I N De Oliveira, T B Dos Santos, FABF De Moura, M L Lyra, and M Serva. Critical behavior of the ideal-gas Bose-Einstein condensation in the Apollonian network. Phys. Rev. E, 88(2):22139, 2013. 
[11] Albert-László Barabási. Scale-free networks: a decade and beyond. Science, 325(5939):412-413, 2009.

[12] Réka Albert and Albert-László Barabási. Statistical mechanics of complex networks. Rev. Mod. Phys., 74(1):47, 2002.

[13] Romualdo Pastor-Satorras and Alessandro Vespignani. Epidemic spreading in scale-free networks. Phys. Rev. Lett., 86(14):3200, 2001.

[14] Zhongzhi Zhang, Shuigeng Zhou, Wenlei Xie, Lichao Chen, Yuan Lin, and Jihong Guan. Standard random walks and trapping on the Koch network with scale-free behavior and small-world effect. Phys. Rev. E, 79(6):61113, 2009.

[15] Reuven Cohen, Daniel Ben-Avraham, and Shlomo Havlin. Percolation critical exponents in scale-free networks. Phys. Rev. E, 66(3):36113, 2002.

[16] M Serva, U L Fulco, and E L Albuquerque. Ising models on the regularized Apollonian network. Phys. Rev. E, 88(4):42823, 2013.

[17] Filippo Radicchi and Santo Fortunato. Explosive percolation in scale-free networks. Phys. Rev. Lett., 103(16):168701, 2009.

[18] Carlos P Herrero. Ising model in scale-free networks: A monte carlo simulation. Phys. Rev. E, 69(6):67109, 2004.

[19] R Burioni, D Cassi, Mario Rasetti, P Sodano, and A Vezzani. Bose-Einstein condensation on inhomogeneous complex networks. J. Phys. B At. Mol. Opt. Phys., 34(23):4697, 2001.

[20] Duncan S Callaway, Mark E J Newman, Steven H Strogatz, and Duncan J Watts. Network robustness and fragility: Percolation on random graphs. Phys. Rev. Lett., 85(25):5468, 2000.

[21] Reuven Cohen, Keren Erez, Daniel Ben-Avraham, and Shlomo Havlin. Resilience of the Internet to random breakdowns. Phys. Rev. Lett., 85(21):4626, 2000.

[22] M Leone, A Vázquez, A Vespignani, and Riccardo Zecchina. Ferromagnetic ordering in graphs with arbitrary degree distribution. Eur. Phys. J. B, 28(2):191-197, 2002.

[23] Xiangwei Chu, Zhongzhi Zhang, Jihong Guan, Shuigeng Zhou, and Mo Li. Different behaviors of epidemic spreading in scale-free networks with identical degree sequence. J. Phys. A Math. Theor., 43(6):65001, 2010.

[24] Yonghui Wu, Xing Li, Zhongzhi Zhang, and Zhihai Rong. The different cooperative behaviors on a kind of scale-free networks with identical degree sequence. Chaos, Solitons and Fractals, 56:91-95, 2013.

[25] Rammal Rammal. Spectrum of harmonic excitations on fractals. J. Phys., 45(2):191-206, 1984.

[26] Oliver Mülken, Maxim Dolgushev, and Mircea Galiceanu. Complex quantum networks: From universal breakdown to optimal transport. Phys. Rev. E, 93(2):22304, 2016.

[27] Maxim Dolgushev, Thomas Guérin, Alexander Blumen, Olivier Bénichou, and Raphaël Voituriez. Contact Kinetics in Fractal Macromolecules. Phys. Rev. Lett., 115(20):208301, 2015.

[28] Chaoming Song, Shlomo Havlin, and Hernán A Makse. Self-similarity of complex networks. Nature, 433(7024):392-395, 2005.

[29] Chaoming Song, Shlomo Havlin, and Hernán A Makse. Origins of fractality in the growth of complex networks. Nat. Phys., 2(4):275-281, 2006.

[30] Albert-László Barabási, Erzsebet Ravasz, and Tamas Vicsek. Deterministic scale-free networks. Phys. A Stat. Mech. its Appl., 299(3):559-564, 2001.

[31] Erzsébet Ravasz, Anna Lisa Somera, Dale A Mongru, Zoltán N Oltvai, and A-L Barabási. Hierarchical organization of modularity in metabolic networks. Science, 297(5586):1551-1555, 2002.

[32] Shlomo Alexander and Raymond Orbach. Density of states on fractals:\{\guillemotleft $\}$ fractons $\{\backslash$ guillemotright $\}$. J. Phys. Lett., 43(17):625-631, 1982.

[33] Rammal Rammal and Gérard Toulouse. Random walks on fractal structures and percolation clusters. J. Phys. Lett., 44(1):13-22, 1983.

[34] Duncan J Watts and Steven H Strogatz. Collective dynamics of small-world'networks. Nature, 393(6684):440-442, 1998.

[35] Zhongzhi Zhang, Shuigeng Zhou, Tao Zou, Lichao Chen, and Jihong Guan. Different thresholds of bond percolation in scale-free networks with identical degree sequence. Phys. Rev. E, 79(3):31110, 2009.

[36] J. C. Slater and G. F. Koster. Simplified LCAO method for the periodic potential problem. Phys. Rev., 94(6):1498-1524, 1954.

[37] Jing Huang and Shuchao Li. ON THE NORMALISED LAPLACIAN SPECTRUM, DEGREE-KIRCHHOFF INDEX AND SPANNING TREES OF GRAPHS. Bull. Aust. Math. Soc., 91(03):353-367, jun 2015.

[38] P. Xie, Y. Lin, and Z. Zhang. Spectrum of walk matrix for Koch network and its application. J. Chem. Phys., 142(22), 2015.

[39] Raffaella Burioni and Davide Cassi. Universal properties of spectral dimension. Phys. Rev. Lett., 76(7):1091, 1996.

[40] Raffaella Burioni and Davide Cassi. Random walks on graphs: ideas, techniques and results. J. Phys. A. Math. Gen., 38(8):R45, 2005.

[41] Davide Cassi. Phase transitions and random walks on graphs: A generalization of the Mermin-Wagner theorem to disordered lattices, fractals, and other discrete structures. Phys. Rev. Lett., 68(24):3631, 1992.

[42] Davide Cassi and Sofia Regina. Spectral dimension of branched structures: universality in geometrical disorder. Phys. Rev. Lett., 70(11):1647, 1993.

[43] Raffaella Burioni, Davide Cassi, and Sofia Regina. Cutting-decimation renormalization for diffusive and vibrational dynamics on fractals. Phys. A Stat. Mech. its Appl., 265(3):323-332, 1999.

[44] Carol K Hall. Scaling in the ideal Bose gas. J. Stat. Phys., 13(2):157-172, 1975. 